in vivo $32: 1381-1386(2018)$

doi:10.21873/invivo.11390

\title{
How Much Ischemia Can the Severely Steatotic Rat Liver Tolerate?
}

\author{
IOANNIS D. KOSTAKIS ${ }^{1,2}$, NIKOLAOS SIKALIAS ${ }^{3}$, KONSTANTINOS ALEXIOU $^{3}$, \\ LAMPRINI MOUNTZALIA $^{3}$, APOSTOLOS PAPALOIS $^{4}$ and THEODORE KARATZAS ${ }^{1,2}$ \\ ${ }^{1}$ Second Department of Propaedeutic Surgery, Laiko General Hospital, \\ National and Kapodistrian University of Athens, Medical School, Athens, Greece; \\ ${ }^{2}$ N.S. Christeas Laboratory of Experimental Surgery and Surgical Research, \\ National and Kapodistrian University of Athens, Medical School, Athens, Greece; \\ ${ }^{3}$ Department of Surgery, Sismanogleion General Hospital, Athens, Greece; \\ ${ }^{4}$ ELPEN Pharmaceuticals Research Center, Pikermi, Greece
}

\begin{abstract}
Aim: Steatotic liver is more susceptible to ischemia-reperfusion injury than is lean liver. Our aim was to investigate the ability of the severely steatotic rat liver to sustain ischemia. Materials and Methods: One hundred male Wistar rats aged 12-14 weeks were included. Fifty rats were given regular diet, while the rest were given a choline-free diet for 12-14 weeks to develop severe liver steatosis. Each group was divided into the following five subgroups: Shamoperated, and 5, 10, 15 and 20 minutes of continuous vascular inflow occlusion. Serum glutamic-oxaloacetic transaminase and serum glutamic-pyruvic transaminase levels were measured at 24 hours postoperatively and the animals were surveilled for 30 days. Results: Serum transaminase levels increased as the duration of ischemia increased in lean livers $(p<0.0001)$, without a significant impact on animal survival. Similarly, serum transaminase levels increased as the duration of ischemia increased in severely steatotic livers $(p<0.0001)$, reaching a plateau after 15 minutes of liver ischemia. Survival was significantly affected after the same cut-off point in rats with steatotic liver $(p<0.0001)$. Serum transaminase levels were greater in severely rats with steatotic liver than in rats with lean liver, when they were adjusted for the duration of liver ischemia. Moreover, survival was reduced when serum transaminase levels surpassed the threshold of 2,000 IU/l $(p<0.0001)$.
\end{abstract}

This article is freely accessible online.

Correspondence to: Ioannis D. Kostakis, MD, M.Sc., Ph.D., 27 Achridos Street, Kato Patissia, 11144, Athens, Greece. Tel: +30 2102288698, Fax: +30 2103813822, e-mail: i.d.kostakis@gmail.com

Key Words: Liver, steatosis, fatty liver disease, rats, ischemia, ischemia-reperfusion injury.
Conclusion: Severely steatotic rat liver can safely tolerate up to 10 minutes of continuous ischemia, with survival being affected after 15 minutes or more. On the other hand, lean rat liver can safely tolerate even 20 minutes of continuous ischemia.

Hepatic steatosis or fatty liver disease has become a common liver pathology, affecting $10-30 \%$ of the general population in most studies worldwide (1-3). The prevalence is similar in autopsy series, with $15-30 \%$ of autopsied cases found to be affected by hepatic steatosis $(1,3)$. Although the exact pathophysiological mechanisms have not been fully elucidated, several risk factors for the development of fatty liver disease have been recognized, such as heavy alcohol consumption, obesity, older age, diabetes mellitus, and dyslipidemia (1-3). The presence of fatty liver disease has been associated with higher rates of postoperative morbidity and mortality after liver resection (4-6). These increased rates of postoperative complications can at least partly be attributed to the occlusion of inflow caused by clamping of the hepatic pedicle (Pringle's maneuver), which is utilized during hepatectomy, along with keeping low central venous pressure in order for blood loss to be reduced, and subsequent ischemia-reperfusion injury $(4,6)$. Although several studies have tried to assess the maximum duration of cumulative ischemia, applied either continuously or intermittently, that the non-diseased or the cirrhotic liver can endure [reviewed in (7)], little is known regarding the ability of steatotic liver to withstand ischemic manipulations. Our aim was to investigate the ability of severely steatotic rat liver to sustain such manipulations. We also tried to identify whether there is an upper limit of duration of liver ischemia after which the survival of rats with severe liver steatosis is affected. Finally, we also attempted to estimate how these rats differ from rats with non-diseased liver. 


\section{Materials and Methods}

Animals. One hundred male Wistar rats aged 12-14 weeks and weighing 250-300 $\mathrm{g}$ were included in this study. Animals were provided by the Pasteur Institute of Athens, Greece. The study was carried out at the Experimental Research Center of ELPEN (Athens, Greece) and was approved by the Veterinary Authority of East Attika Prefecture (Protocol reference numbers: 1633 and 2659, directive 609/1986) and performed complying with the rules of experimentation and 3Rs (Replace, Reduce \& Refine). There was free access to water and food, constant ambient temperature and a cycle of alternating light and dark every 12 hours. The 100 rats were randomly divided into two large groups, each comprised of 50 rats. The first group (lean liver, L) was provided with a regular diet, while the second one (steatotic liver, $S$ ) was given a choline-free diet (PF1877, Mucedola; Settimo Milanese, Milan, Italy) for 12-14 weeks in order to develop fatty infiltration of the liver exceeding $60 \%$ of the hepatic parenchyma, as previous studies have shown (813). After a period of 12-14 weeks of feeding, all rats were aged 24-26 weeks and had reached a mean body weight of $401 \pm 23 \mathrm{~g}$ for the first group and $497 \pm 54 \mathrm{~g}$ for the second group. At this point, each group of animals was further subdivided randomly into five subgroups as follows.

Groups L1 and S1 (n=6): Sham operated rats; groups L2 and S2 $(n=11)$ : rats which underwent 5 minutes of continuous liver ischemia; groups L3 and S3 $(n=11)$ : rats which underwent 10 minutes of continuous liver ischemia; groups L4 and S4 $(n=11)$ : rats underwent 15 minutes of continuous liver ischemia; groups L5 and S5 $(n=11)$ : rats which underwent 20 minutes of continuous liver ischemia.

Surgical procedures. All animals were anesthetized using isoflurane, intubated and ventilated mechanically. Afterwards, their abdominal wall was sterilized and a median laparotomy incision was made. In the sham-operated rats (L1 and S1 groups), the liver was mobilized only, no further manipulations were applied, the abdominal wall was closed and the animals regained consciousness. All the other animals (groups L2-L5 and S2-S5) were subjected to continuous vascular occlusion in the common perfusion area of the two right hepatic lobes with Rummel's technique (14), which lasted according to the interval stated above for each group. After vascular occlusion was reversed, the abdominal wall was closed and the animals regained consciousness.

Postoperative surveillance. Blood samples $(1.5 \mathrm{ml})$ were drawn from the tail of each rat at 24 hours after the operation, and centrifuged at $3,500 \times g$ for 10 minutes. An enzymatic method (Advia 1800 Chemistry Analyzer system, Siemens, Munich, Germany) was used for measurement of serum glutamic-oxaloacetic transaminase (SGOT) and serum glutamic-pyruvic transaminase (SGPT) levels with reagent kits (SGOT/1417-0070, SGPT/14170080) provided by Medicon Hellas (Athens, Greece). All animals followed the previously administered diet postoperatively, and were surveilled for 30 days after the operation; deaths within this time period were noted.

Statistical analysis. The Shapiro-Wilk test was used for the assessment of normality of data distribution. Comparisons between two groups were made using Student's $t$-test, Welch's $t$-test or Mann-Whitney $U$-test, as appropriate. Comparisons among three or more groups were made using analysis of variance (ANOVA) or
Table I. Serum transaminase levels at 24 hours postoperatively in rats with lean liver (L groups) and those with steatotic liver (S groups) after sham operation (L1/S1) and after 5, 10, 15 and 20 minutes of continuous occlusion of vascular inflow (L2-5/S2-5, respectively).

\begin{tabular}{|c|c|c|c|}
\hline Transaminase & L1 & S1 & $p$-Value \\
\hline \multicolumn{4}{|l|}{ SGOT (IU/l) } \\
\hline Mean \pm SD & $53.5 \pm 9.6$ & $69.7 \pm 8$ & 0.016 \\
\hline Median (min-max) & $51.5(43-71)$ & $68.5(59-83)$ & \\
\hline \multicolumn{4}{|l|}{ SGPT (IU/1) } \\
\hline Mean \pm SD & $53.2 \pm 10.7$ & $70.5 \pm 9.4$ & 0.021 \\
\hline \multirow[t]{2}{*}{ Median (min-max) } & $52.5(39-71)$ & $69.5(59-86)$ & \\
\hline & L2 & $\mathrm{S} 2$ & $p$-Value \\
\hline \multicolumn{4}{|l|}{ SGOT (IU/l) } \\
\hline Mean \pm SD & $169.6 \pm 15.1$ & $255.1 \pm 28.5$ & $<0.0001$ \\
\hline Median (min-max) & $169(148-193)$ & $248(218-312)$ & \\
\hline \multicolumn{4}{|l|}{ SGPT (IU/1) } \\
\hline Mean \pm SD & $147.2 \pm 15$ & $228.2 \pm 39.8$ & $<0.0001$ \\
\hline \multirow[t]{2}{*}{ Median (min-max) } & $153(121-168)$ & $212(168-284)$ & \\
\hline & L3 & S3 & $p$-Value \\
\hline \multicolumn{4}{|l|}{ SGOT (IU/1) } \\
\hline Mean \pm SD & $389.1 \pm 32.7$ & $502.5 \pm 42.2$ & $<0.0001$ \\
\hline Median (min-max) & $385(326-429)$ & $506(407-554)$ & \\
\hline \multicolumn{4}{|l|}{ SGPT (IU/1) } \\
\hline Mean \pm SD & $317.6 \pm 51.9$ & $460.5 \pm 53.4$ & $<0.0001$ \\
\hline \multirow[t]{2}{*}{ Median (min-max) } & $302(235-396)$ & $453(368-551)$ & \\
\hline & L4 & S4 & $p$-Value \\
\hline \multicolumn{4}{|l|}{ SGOT (IU/l) } \\
\hline Mean \pm SD & $620.9 \pm 52.3$ & $2676.5 \pm 1080$ & $<0.0001$ \\
\hline Median (min-max) & $607(539-704)$ & $3238(1127-3732)$ & \\
\hline \multicolumn{4}{|l|}{ SGPT (IU/l) } \\
\hline Mean \pm SD & $521.8 \pm 79.5$ & $2317.9 \pm 1034.8$ & $<0.0001$ \\
\hline \multirow[t]{2}{*}{ Median (min-max) } & $492(416-650)$ & $2974(886-3318)$ & \\
\hline & L5 & S5 & $p$-Value \\
\hline \multicolumn{4}{|l|}{ SGOT (IU/l) } \\
\hline Mean \pm SD & $1208.8 \pm 130.5$ & $3212 \pm 1353$ & 0.001 \\
\hline Median (min-max) & $1193(996-1452)$ & $3281(1649-6738)$ & \\
\hline \multicolumn{4}{|l|}{ SGPT (IU/1) } \\
\hline Mean \pm SD & $1185.4 \pm 115.6$ & $2972.9 \pm 1393.6$ & 0.002 \\
\hline Median (min-max) & $1190(1010-1327)$ & $2894(1310-6693)$ & \\
\hline$p$-Value & $<0.0001$ & $<0.0001$ & \\
\hline
\end{tabular}

Kruskal-Wallis test, as appropriate. Correlations between quantitative parameters were tested with Spearman's rank correlation coefficient. Kaplan-Meier curves and log-rank test were used for survival comparisons among different groups. All tests were two-tailed and results were considered statistically significant when the $p$-value was less than 0.05. The Statistical Package for Social Sciences (23rd edition; IBM Corporation, Armonk, NY, USA) was used for statistical analysis. 


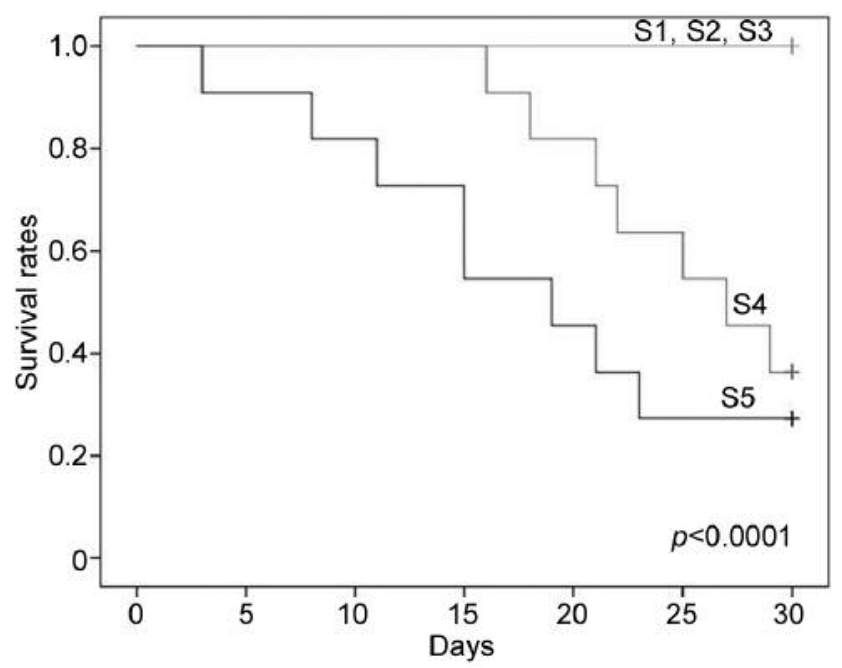

Figure 1. Survival according to duration of liver ischemia in rats with severely steatotic liver. S1: Sham operation; S2-S5, ischemia of 5, 10, 15 and 20 minutes, respectively.

\section{Results}

Correlation between serum SGOT and SGPT levels. There was a high positive correlation between serum SGOT and SGPT levels at 24 hours postoperatively when considering all animals together $(\mathrm{r}=0.988, p<0.0001)$, as well as when considering rats with lean liver $(\mathrm{r}=0.978, p<0.0001)$ and rats with steatotic liver $(\mathrm{r}=0.981, p<0.0001)$ separately.

Serum transaminase levels in rats with lean liver according to duration of liver ischemia. There was a steady increase in serum SGOT and SGPT levels at 24 hours after the operation as the duration of liver ischemia increased $(p<0.0001)$. In particular, individual groups differed highly significantly when they were compared with the rest of the groups $(p<0.0001$, for both transaminases). Serum SGOT and SGPT levels in each group of rats with lean liver are shown in Table I. There were no differences concerning survival, since all rats with lean liver survived up to 30 days, which was the endpoint of our study.

Serum transaminase levels in rats with severely steatotic liver according to duration of liver ischemia. There was a steady increase in serum SGOT and SGPT levels at 24 hours after the operation as the duration of liver ischemia increased $(p<0.0001)$. In particular, individual groups differed highly significantly when they were compared with the rest of the groups for SGOT $(p<0.001)$ and for SGPT $(p<0.002)$. The only exception to this was the comparison between S4 and S5 groups (SGOT: $p=0.974$, SGPT: $p=0.914)$. Serum SGOT and SGPT levels in each group of rats with steatotic liver are shown in Table I.

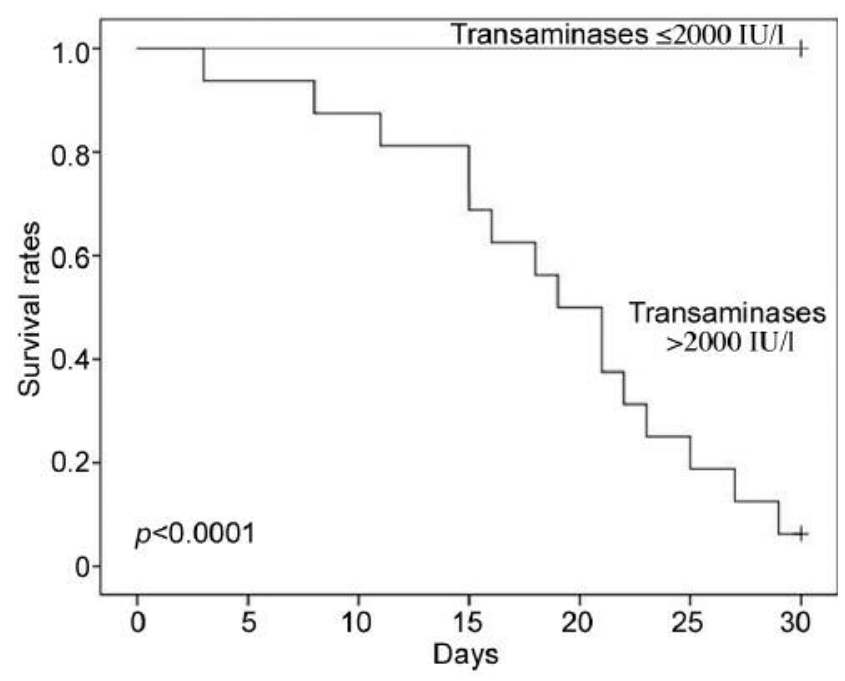

Figure 2. Survival according to serum transaminase levels in rats with severely steatotic liver.

Furthermore, there was a significant difference regarding survival $(p<0.0001)$ and 15 rats with steatotic liver had died by the end of our study $(15 / 50 ; 30 \%)$. No death was noted in groups S1, S2 and S3 during the 30 days of our study. On the contrary, seven out of the 11 animals $(63.6 \%)$ in the $\mathrm{S} 4$ group and eight out of the 11 animals $(72.7 \%)$ in the S5 group had died before the 30-day endpoint of our study (Figure 1). Another interesting finding was the fact that all steatotic rats with serum transaminase (SGOT or SGPT) levels higher than $2000 \mathrm{IU} / 1$ at 24 hours postoperatively except one (15/16; $93.75 \%$ ) died before the endpoint of 30 days. On the other hand, all steatotic rats with serum transaminase levels of 2000 IU/I or lower at 24 hours postoperatively remained alive until the endpoint of our study ( $p<0.0001$ ) (Figure 2$)$. This cut-off point of $2000 \mathrm{IU} / 1$ was not applied to rats with lean liver because no such rat died within the 30 days of surveillance and at 24 hours postoperatively, serum transaminase levels were not higher than $1500 \mathrm{IU} / 1$ in these animals.

Comparison between rats with lean liver and those with severely steatotic liver. Comparisons were made between lean rats and rats with steatotic liver by interval of liver ischemia. Serum SGOT and SGPT levels were significantly higher at 24 hours postoperatively in animals with steatotic liver when compared with animals with lean liver for each interval of liver ischemia. The results of each comparison are listed in Table I.

As far as survival is concerned, there were no differences when we compared group L1 with S1, L2 with S2, and L3 with $\mathrm{S} 3$, because no animal had died in these six groups before the endpoint of 30 days. On the contrary, the fact that 


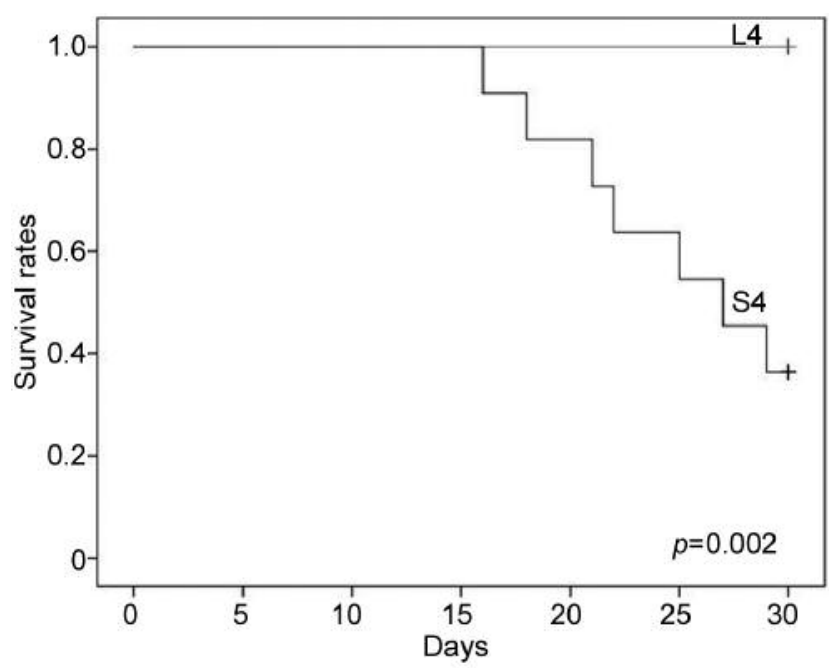

Figure 3. Comparison of survival between groups L4 (lean liver, 15 minutes of liver ischemia) and S4 (steatotic liver, 15 minutes of liver ischemia).

there were no deaths in L4 and L5 groups, but there were seven and eight deaths in S4 and S5 groups, respectively, within the study period of 30 days, led to significant differences in survival when comparing group L4 with S4 $(p=0.002)$ (Figure 3$)$ and L5 with S5 ( $p=0.0004)$ (Figure 4).

\section{Discussion}

Many studies have tried to assess the underlying pathophysiological mechanisms of ischemia-reperfusion injury in liver. It is obvious that not only ischemia, but also reperfusion has deleterious effects on liver tissue. Several histological alterations in liver have been observed during this process whose consequence is microcirculatory failure, such as swelling of Kupffer and endothelial cells, platelet aggregation, leukocyte infiltration and vasoconstriction. Liver injury intensifies due to the additional release of free radicals and inflammatory cytokines, such as interleukins and tumor necrosis factor, from leukocytes and Kupffer cells $(15,16)$ during hepatic ischemia/reperfusion. The response of liver to ischemia-reperfusion injury is further divided into two phases, an early and a late one. The early phase is characterized by the activation of Kupffer cells, which release cytokines and free radicals, while the late phase is characterized by the infiltration of neutrophils into liver parenchyma, which release cytokines, proteases and free radicals (15). In fatty liver disease, mitochondrial dysfunction and altered signal transduction in several pathways lead to increased production of reactive oxygen species, which is further aggravated during reperfusion, rendering steatotic livers more susceptible to damage from ischemia-reperfusion injury $(6,17)$.

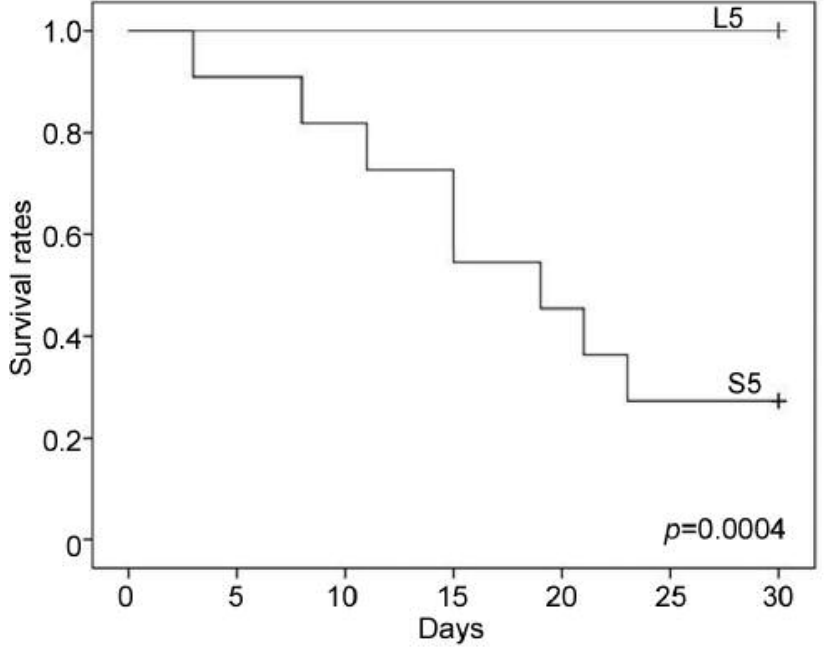

Figure 4. Comparison of survival between groups L5 (lean liver, 20 minutes of liver ischemia) and S5 (steatotic liver, 20 minutes of liver ischemia).

Many studies have aimed to estimate the longest duration of ischemia that diseased and non-diseased liver can tolerate. There is much evidence showing that the liver can sustain longer periods of intermittent rather than continuous occlusion of vascular inflow; the intermediate periods of perfusion between the periods of ischemia allow the liver to tolerate ischemia for a longer duration in total (7). Similarly, ischemic preconditioning, which refers to the application of short intervals of ischemia prior to longer sustained periods of ischemia, reduces cell death and inflammatory response, along with improved microcirculation in non-diseased and steatotic livers (18-20). Healthy human livers can endure continuous occlusion of vascular inflow for up to 90 minutes, whereas well-selected cirrhotic human livers can only withstand for up to 50-60 minutes. However, the application of intermittent occlusion of vascular inflow can extend the total duration of ischemia to up to 300 minutes for healthy human livers and up to 200 minutes for wellselected cirrhotic human livers (7).

However, little is known concerning the duration of ischemia that steatotic livers are able to tolerate without increasing postoperative morbidity and mortality. A few studies have compared the postoperative survival of rats with lean liver with that of rats with severely steatotic liver (fatty infiltration of liver exceeding $60 \%$ of hepatic parenchyma) after subjecting them to total hepatic ischemia for 60 minutes with subsequent reperfusion (21-23). The 7-day postoperative survival rates ranged between $70 \%$ and $100 \%$ for rats with lean liver, while they were lower for rats with severely steatotic liver, ranging between $0 \%$ and 64\% (21-23). However, this difference was smaller when both types of rats 
underwent 30 minutes of total hepatic ischemia followed by reperfusion (rats with lean liver: $100 \%$, rats with severe hepatic steatosis: $75-95 \%)(22,23)$. Postoperative serum transaminase levels were also higher in rats with severe hepatic steatosis than in rats with lean liver at various time intervals after liver ischemia-reperfusion $(21,22,24,25)$.

A technique that has been utilized in many animal studies for the amelioration of ischemia-reperfusion injury is ischemic preconditioning of the liver. This means that a brief period of ischemia is applied to the liver prior to a period of prolonged ischemia, which leads to milder tissue damage and better postoperative hepatic function $(13,18-20)$. This tactic has also been tested in rats with severe hepatic steatosis (fatty infiltration of liver exceeding $60 \%$ of hepatic parenchyma) in a few studies. As far as postoperative survival is concerned, the application of hepatic ischemia and reperfusion for 5 and 10 minutes, respectively, before subjecting rats with steatotic liver to hepatic ischemia for 60 minutes resulted in a great increase of 30-day survival rate from $0-70 \%(26,27)$. Postoperative serum transaminase levels were also lower in rats with severe hepatic steatosis that had undergone ischemic preconditioning before prolonged hepatic ischemia than in those without ischemic preconditioning at various time intervals after operation (25-31).

In our study, we tried to assess the extent of ischemiareperfusion injury by measuring serum transaminase levels as markers of hepatic cell death, and to identify whether there is an upper limit of liver ischemia duration after which the survival of rats is affected. We studied the effects of different durations of continuous occlusion of vascular inflow in both lean and severely steatotic livers. According to our findings, liver damage increased as the duration of ischemia increased in rats with lean livers, but without having any impact on their survival. On the contrary, liver damage increased as the duration of ischemia increased in rats with severely steatotic livers, but reached a plateau after 15 minutes of continuous occlusion. Survival was also found to be affected after the same cut-off point in rats with steatotic liver. Another interesting finding is the fact that liver damage was greater in rats with severely steatotic liver than in those with lean liver, when adjusted for the duration of liver ischemia. Moreover, it was observed that the survival was affected when serum transaminase levels at 24 hours postoperatively surpassed the threshold of 2,000 IU/I.

In conclusion, severely steatotic rat liver can safely tolerate up to 10 minutes of continuous ischemia, whereas survival is affected after 15 or more minutes of continuous ischemia. On the other hand, lean rat liver can safely tolerate even 20 minutes of continuous ischemia. These findings suggest that severely steatotic liver is more susceptible to ischemia and subsequent ischemia-reperfusion injury when compared with lean liver. Therefore, ischemic manipulations should be applied with consideration for cases with severely steatotic liver.

\section{Conflicts of Interest}

There are no conflicts of interest to declare in regard to this study.

\section{Acknowledgements}

This study was funded by Scholarship - Grant by the Experimental Research Center of ELPEN Pharmaceuticals (E.R.C.E).

\section{References}

1 Vernon G, Baranova A and Younossi ZM: Systematic review: The epidemiology and natural history of non-alcoholic fatty liver disease and non-alcoholic steatohepatitis in adults. Aliment Pharmacol Ther 34: 274-285, 2011.

2 Bellentani S: The epidemiology of non-alcoholic fatty liver disease. Liver Int 37: 81-84, 2017.

3 Sayiner M, Koenig A, Henry L and Younossi ZM: Epidemiology of nonalcoholic fatty liver disease and nonalcoholic steatohepatitis in the United States and the rest of the world. Clin Liver Dis 20: 205-214, 2016.

4 Veteläinen R, van Vliet A, Gouma DJ and van Gulik TM: Steatosis as a risk factor in liver surgery. Ann Surg 245: 20-30, 2007.

5 de Meijer VE, Kalish BT, Puder $M$ and Ijzermans JN: Systematic review and meta-analysis of steatosis as a risk factor in major hepatic resection. Br J Surg 97: 1331-1339, 2010.

6 Tashiro H, Kuroda S, Mikuriya Y and Ohdan H: Ischemiareperfusion injury in patients with fatty liver and the clinical impact of steatotic liver on hepatic surgery. Surg Today 44: 1611-1625, 2014.

7 van Riel WG, van Golen RF, Reiniers MJ, Heger M and van Gulik TM: How much ischemia can the liver tolerate during resection? Hepatobiliary Surg Nutr 5: 58-71, 2016.

8 Veteläinen R, van Vliet AK and van Gulik TM: Severe steatosis increases hepatocellular injury and impairs liver regeneration in a rat model of partial hepatectomy. Ann Surg 245: 44-50, 2007.

9 Mendes-Braz M, Elias-Miró M, Jiménez-Castro MB, CasillasRamírez A, Ramalho FS and Peralta C: The current state of knowledge of hepatic ischemia-reperfusion injury based on its study in experimental models. J Biomed Biotechnol 2012: 298657, 2012.

10 Veteläinen R, van Vliet A and van Gulik TM: Essential pathogenic and metabolic differences in steatosis induced by choline or methione-choline deficient diets in a rat model. J Gastroenterol Hepatol 22: 1526-1533, 2007.

11 Koteish A and Diehl AM: Animal models of steatosis. Semin Liver Dis 21: 89-104, 2001.

12 Anstee QM and Goldin RD: Mouse models in non-alcoholic fatty liver disease and steatohepatitis research. Int J Exp Pathol 87: 1-16, 2006.

13 Jiang Y, Tang JJ, Wu BQ, Yuan B and Qu Z: The protective effects of different-time-ischemic preconditioning on the reperfusion injury in fatty livers in rats. PLoS One 8: e58086, 2013.

14 Chu MJ, Hickey AJ, Phillips AR and Bartlett AS: The impact of hepatic steatosis on hepatic ischemia-reperfusion injury in experimental studies: A systematic review. Biomed Res Int 2013: 192029, 2013. 
15 Konishi $\mathrm{T}$ and Lentsch $\mathrm{AB}$ : Hepatic ischemia/reperfusion: mechanisms of tissue injury, repair, and regeneration. Gene Expr 17: 277-287, 2017

16 Saidi RF and Kenari SK: Liver ischemia/reperfusion injury: an overview. J Invest Surg 27: 366-379, 2014.

17 Reiniers MJ, van Golen RF, van Gulik TM and Heger M: Reactive oxygen and nitrogen species in steatotic hepatocytes: a molecular perspective on the pathophysiology of ischemiareperfusion injury in the fatty liver. Antioxid Redox Signal 21: 1119-1142, 2014

18 Robertson FP, Fuller BJ and Davidson BR: An evaluation of ischaemic preconditioning as a method of reducing ischaemia reperfusion injury in liver surgery and transplantation. J Clin Med 6: E69, 2017.

19 Chu MJ, Vather R, Hickey AJ, Phillips AR and Bartlett AS: Impact of ischaemic preconditioning on experimental steatotic livers following hepatic ischaemia-reperfusion injury: a systematic review. HPB 17: 1-10, 2015.

20 Sikalias N, Karatzas T, Alexiou K, Mountzalia L, Demonakou M, Kostakis ID, Zacharioudaki A, Papalois A and Kouraklis G: Intermittent ischemic preconditioning protects against hepatic ischemia-reperfusion injury and extensive hepatectomy in steatotic rat liver. J Invest Surg Jun 23: 1-12, 2017.

21 Caraceni P, Nardo B, Domenicali M, Turi P, Vici M, Simoncini M, De Maria N, Trevisani F, Van Thiel DH, Derenzini M, Cavallari A and Bernardi M: Ischemia-reperfusion injury in rat fatty liver: Role of nutritional status. Hepatology 29: 1139-1146, 1999.

22 Hakamada K, Sasaki M, Takahashi K, Umehara Y and Konn M: Sinusoidal flow block after warm ischemia in rats with dietinduced fatty liver. J Surg Res 70: 12-20, 1997.

23 Hui AM, Kawasaki S, Makuuchi M, Nakayama J, Ikegami T and Miyagawa S: Liver injury following normothermic ischemia in steatotic rat liver. Hepatology 20: 1287-1293, 1994.

24 Marsman HA, Heger M, Kloek JJ, Nienhuis SL, ten Kate FJ and van Gulik TM: Omega-3 fatty acids reduce hepatic steatosis and consequently attenuate ischemia-reperfusion injury following partial hepatectomy in rats. Dig Liver Dis 43: 984-990, 2011.
25 Rolo AP, Teodoro JS, Peralta C, Rosello-Catafau J and Palmeira $\mathrm{CM}$ : Prevention of $\mathrm{I} / \mathrm{R}$ injury in fatty livers by ischemic preconditioning is associated with increased mitochondrial tolerance: The key role of ATP synthase and mitochondrial permeability transition. Transpl Int 22: 1081-1090, 2009.

26 Serafín A, Roselló-Catafau J, Prats N, Xaus C, Gelpí E and Peralta C: Ischemic preconditioning increases the tolerance of fatty liver to hepatic ischemia-reperfusion injury in the rat. Am J Pathol 161: 587-601, 2002.

27 Serafín A, Roselló-Catafau J, Prats N, Gelpí E, Rodés J and Peralta C: Ischemic preconditioning affects interleukin release in fatty livers of rats undergoing ischemia/reperfusion. Hepatology 39: 688-698, 2004.

28 Tacchini L, Cairo G, De Ponti C, Massip M, Rosellò-Catafau J and Peralta C: Up regulation of IL- 6 by ischemic preconditioning in normal and fatty rat livers: association with reduction of oxidative stress. Free Radic Res 40: 1206-1217, 2006.

29 Casillas-Ramirez A, Amine-Zaouali M, Massip-Salcedo M, Padrissa-Altés S, Bintanel-Morcillo M, Ramalho F, Serafín A, Rimola A, Arroyo V, Rodés J, Roselló-Catafau J and Peralta C: Inhibition of angiotensin II action protects rat steatotic livers against ischemia-reperfusion injury. Crit Care Med 36: 1256-1266, 2008.

30 Massip-Salcedo M, Zaouali MA, Padrissa-Altés S, CasillasRamirez A, Rodés J, Roselló-Catafau J and Peralta C: Activation of peroxisome proliferator-activated receptor-alpha inhibits the injurious effects of adiponectin in rat steatotic liver undergoing ischemia-reperfusion. Hepatology 47: 461-472, 2008.

31 Massip-Salcedo M, Casillas-Ramirez A, Franco-Gou R, Bartrons R, Ben Mosbah I, Serafin A, Roselló-Catafau J and Peralta C: Heat-shock proteins and mitogen-activated protein kinases in steatotic livers undergoing ischemia-reperfusion: Some answers. Am J Pathol 168: 1474-1485, 2006.

Received July 182018

Revised September 22, 2018

Accepted September 25, 2018 